\title{
DW-MRI for esophageal squamous cell carcinoma, correlations between ADC values with histologic differentiation and VEGF expression: A retrospective study
}

\author{
QINGXUE CONG ${ }^{1,2},{\text { GUANG } \mathrm{LI}^{2} \text {, YONGFENG WANG }}^{3}$, SHANGUO ZHANG $^{4}$ and HAO ZHANG ${ }^{5}$ \\ ${ }^{1}$ Department of Radiotherapy, Daqing Longnan Hospital, Daqing, Heilongjiang 163453; Departments of ${ }^{2}$ Radiotherapy and \\ ${ }^{3}$ Radiology, The First Affiliated Hospital of China Medical University, Shenyang, Liaoning 110001; \\ Departments of ${ }^{4}$ Radiology and ${ }^{5}$ Oncology, Daqing Longnan Hospital, Daqing, Heilongjiang 163453, P.R. China
}

Received November 27, 2017; Accepted September 4, 2018

DOI: $10.3892 / 01.2019 .9934$

\begin{abstract}
The aim of the present study was to assess the correlations between diffusion-weighted magnetic resonance imaging (DW-MRI) features with the histologic differentiation and the expression of vascular endothelial growth factor (VEGF) in esophageal squamous cell carcinoma (ESCC). A total of 52 patients with ESCC included in the present study received radiotherapy, and all patients underwent contrast enhanced MRI and DW-MRI prior to and following radiotherapy. The diffusion sensitivity coefficient ( $b$ value) was set as $800 \mathrm{~s} / \mathrm{mm}^{2}$. Apparent diffusion coefficient (ADC) values were automatically computed. VEGF expression was evaluated by immunohistochemical staining. The results demonstrated that the pathological grading of ESCC was positively correlated with ADC values $(r=0.635, \mathrm{P}=0.0007)$, and the VEGF expression was inversely correlated with ADC values $(r=-0.321, \mathrm{P}=0.008)$. However, no correlation was identified between the pathological grading and the VEGF expression $(r=0.178, \mathrm{P}=0.284)$. All patients were categorized as complete response (CR) or partial response (PR) and the ADC values were increased significantly following radiotherapy. The mean ADC values in the $\mathrm{CR}$ group were higher than the PR group prior to radiotherapy $(t=5.156, \mathrm{P}=0.0004)$. Therefore, we concluded that the DWI with ADC value measurement may represent the grade of tumor histologic differentiation and the degree of VEGF expression, and may also serve as a useful marker to predict radiotherapy and anti-VEGF response in ESCC. ADC value may be a substitution for assessing tumor
\end{abstract}

Correspondence to: Dr Guang Li, Department of Radiotherapy, The First Affiliated Hospital of China Medical University, 155 Nanjing North Street, Shenyang, Liaoning 110001, P.R. China E-mail: congqxcmu@163.com

Key words: esophageal squamous cell carcinoma, diffusion-weighted magnetic resonance imaging, apparent diffusion coefficient, histologic differentiation angiogenesis and novel prognostic factor and contribute to the treatment of ESCC.

\section{Introduction}

Esophageal squamous cell carcinoma (ESCC) is a frequently occurring maligantnt tumor of the digestive system (1). Radiotherapy is the principal treatment for advanced ESCC (2). Clinically, enhancement computed tomography (CT), contrast enhanced magnetic resonance imaging (MRI) and barium meal is the main methods for evaluating the lesion and therapeutic effects (3). However, these examinations are judged by anatomical morphological changes, and lack the ability to display the microscopic pathology and physiological status of the tumor (4).

Diffusion-weighted magnetic resonance imaging (DW-MRI) can noninvasively observe the movement of water molecules in living tissues and provide pathological and physiological changes in vivo, which can be used to diagnose and evaluate the therapeutic effect at the cellular level in the early stage of disease $(5,6)$. Tumor cells have been demonstrated to proliferate vigorously and be arranged tightly; therefore, the movement of water molecules decreases sharply inside and outside cells (7). Consequently, the apparent diffusion coefficient (ADC) value of tumor tissue was lower than that of normal tissue, and the signal of DW-MRI was markedly higher than that of normal tissue, and the diffusion was limited (8). DW-MRI is a useful supplement to conventional imaging evaluation, and has recently been used in radiotherapy target delineation and efficacy evaluation of esophageal cancer (9).

Tumor growth, metastasis and invasion require numerous new blood vessels to provide nutrients and discharge metabolic waste (10). Vascular endothelial growth factor (VEGF) can promote the proliferation and migration of vascular endothelial cells, and is one of the factors that affect the angiogenesis of tumors (11). Jiang et al (11) demonstrated that VEGF protein expression was significantly greater in esophageal cancer than normal epithelial tissue. In addition, VEGF protein expression of the high microvessel density (MVD) group was significantly lower than in the low MVD group with relation to clinical pathological 
staging, differentiation and lymph node metastasis (12). VEGF might make a valuable index of recrudesce and treatment of tumor in clinic, and also a useful marker to predict anti-VEGF treatment response, VEGF is a leading factor of tumor angiogenesis, the anti-angiogenesis therapy aimed at VEGF has probably provided a new chance to malignant tumor treatment (13).

In the present retrospective study, samples collected from 52 patients with pathologically proven ESCC from the First Affiliated Hospital of China Medical University were analyzed. DW-MRI was used to measure the ADC value of tumors, and the correlations between ADC value and VEGF expression and pathological grade of ESCC were observed. To explore the value of DW-MRI in the metabolism, proliferation, efficacy evaluation and prognosis evaluation of ESCC, so as to guide clinical treatment.

\section{Patients and methods}

Patient selection. From March 2014 to January 2016, a total of 52 patients with pathologically proven ESCC from the First Affiliated Hospital of China Medical University were collected. The set of standard for: Random selection, cases perfect follow-up, all patients were at an advanced stage of ESCC, which was confirmed by pathology and clinically, No radiotherapy, chemotherapy and interventional therapy were performed prior MRI examination, No contraindications for MRI examination. The patients were aged between 47-80 years, with a median age of 65 years, and the cohort comprised of 32 males and 20 females. There were 10 well-differentiated cancers, 28 moderately differentiated ones and 14 poorly differentiated ones (14). All patients received standard intensity modulated radiation therapy. The above cases were confirmed by the ethics committee of the First Affiliated Hospital of China Medical University who also provided study approval and patients provided written, informed consent.

MRI device and scanning sequence and parameters. All MRIs were performed by a 3.0T system (GE Medical System, Signa Excite HD) with 8-channel phased-array coil and GE ADW4.4 workstation. MRI examinations were performed prior to and following radiotherapy for 4-6 weeks. The MRI protocols included the T1-weighted (T1W), T2-weighted (T2W), and DWI. All patients were imaged in supine headfirst position. Sagittal T2W SE sequence: Repetition time (TR): $1750 \mathrm{~ms}$, echo time (TE): $98.0 \mathrm{~ms}$, field of view (FOV): $340 \mathrm{~mm}$ section thickness: $4 \mathrm{~mm}$, space: $1 \mathrm{~mm}$, matrix: $512 \times 512$ were performed. Axial T2W sequences with fat suppression: TR: 7500 ms, TE: 102 ms, FOV: 380 mm, section thickness: 4 mm, space: $1 \mathrm{~mm}$, matrix: 512x384 were performed. Axial T1W GR sequences: TR: $230 \mathrm{~ms}$, TE: $3.15 \mathrm{~ms}$, FOV: $400 \mathrm{~mm}$, section thickness: $4 \mathrm{~mm}$, space: $1 \mathrm{~mm}$, matrix: $512 \times 512$ were performed. Axial DWI was performed with single-shot SE-EPI sequences and b values, 0 and $800 \mathrm{~s} / \mathrm{mm}^{2}$, TR: $7500 \mathrm{~ms}$, TE: $65.0 \mathrm{~ms}$, FOV: $380 \mathrm{~mm}$, section thickness: $4 \mathrm{~mm}$, space: $1 \mathrm{~mm}$ were performed. ADC maps were generated with commercially available software GE ADW4.4 workstation system.

MRI image analysis and processing. Two radiologists with 5 years of abdominal MRI experience reviewed the MRI images independently. The ADC value was automatically calculated by a computer program included in the GE ADW4.4 workstation Functool software (GE Healthcare). The region with the most clearly displayed lesion was set as the region of interest (ROIs). Liquefaction, cystic change and necrosis of tumors were avoided when the ROIs were established. The ADC values of each ROI were measured 3 times, and the mean ADC value was set as the final value.

VEGF expression was evaluated by immunohistochemical staining. All the slices underwent conventional dewaxing and hydration processes at room temperature using xylene $0.5 \mathrm{~h}$, $100 \%$ alcohol for $0.5 \mathrm{~h}, 100 \%$ alcohol for $1.5 \mathrm{~h}, 95 \%$ alcohol for $1.5 \mathrm{~h}, 90 \%$ alcohol for $1 \mathrm{~h} 80 \%$ alcohol for $1 \mathrm{~h}$ and finally $70 \%$ alcohol for $0.5 \mathrm{~h}$; and were treated with $3 \% \mathrm{H}_{2} \mathrm{O}_{2}$ solution at room temperature for $10 \mathrm{~min}$. After sealing solution, rabbit anti-rat VEGF polyclonal antibody (cat. no. ab1316; 1:100, Abcam, Cambridge, MA, USA) were added. Then all the slices were placed at $4^{\circ} \mathrm{C}$ overnight and were incubated with goat anti-rabbit IgG (ab187910, 1:1,000, Abcam, Cambridge, UK) secondary antibody at $37^{\circ} \mathrm{C}$ for $30 \mathrm{~min}$. Visualization was performed by $\mathrm{DAB}$ solution (30 $\mathrm{min}$ at room temperature) and re-dyeing was finished with hematoxylin (30 $\mathrm{min}$ at room temperature). The negative control group used PBS buffer instead of the primary antibody. A total of 5 fields of view under high power light microscopy (at magnification, x100) were randomly selected from each sample and the cells in which the cytoplasm was stained brown or yellow were considered as expression-positive cells. The percentage of positive cells in total cells was then calculated. Percentage of positive cells=(number of positive cells/total cells) $\mathrm{x} 100 \%$. VEGF is principally expressed in cytoplasm or cell membrane of tumor cells, and weakly positive in tumor vascular endothelial cells and hepatic sinusoidal endothelial cells. Criteria: Negative (-), staining cell number $<5 \%$, weak positive $(+)$ : Between 5 and $25 \%$, positive $(++)$ : Between 26 and $50 \%$, strong positive $(+++):>50 \%$.

The changes of ADC value prior to and following radiotherapy of ESCC. MRI examinations were performed prior to and following radiotherapy for 4-6 weeks, and the treatment response was evaluated. The mean ADC values were measured separately. The correlation between ADC value and VEGF expression and pathological grade of ESCC was observed, and the change of ADC value of esophageal lesions prior to and following radiotherapy were compared.

Statistical analysis. SPSS 17.0 statistics software (SPSS, Inc., Chicago, IL, USA) was employed to perform statistical analysis. Experiments were performed three times and data are expressed as mean \pm SD. If multiple sets of variables were consistent with homogeneity of variance, one-way analysis of variance (ANOVA) was for multi-group comparisons, followed by LSD post-hoc test. The mean ADC values were tested by homogeneity of variance. LSD post-hoc test was used to compare between VEGF expression groups and pathological grade groups. Homogeneity test of variance was used to compare ADC values and VEGF expression. Kruskal-Wallis H test was used to compare VEGF expression and pathological grading. The Pearson's correlation test was used to correlate between ADC values and VEGF expression and pathological grading. The degree of correlation was classified as a direct 


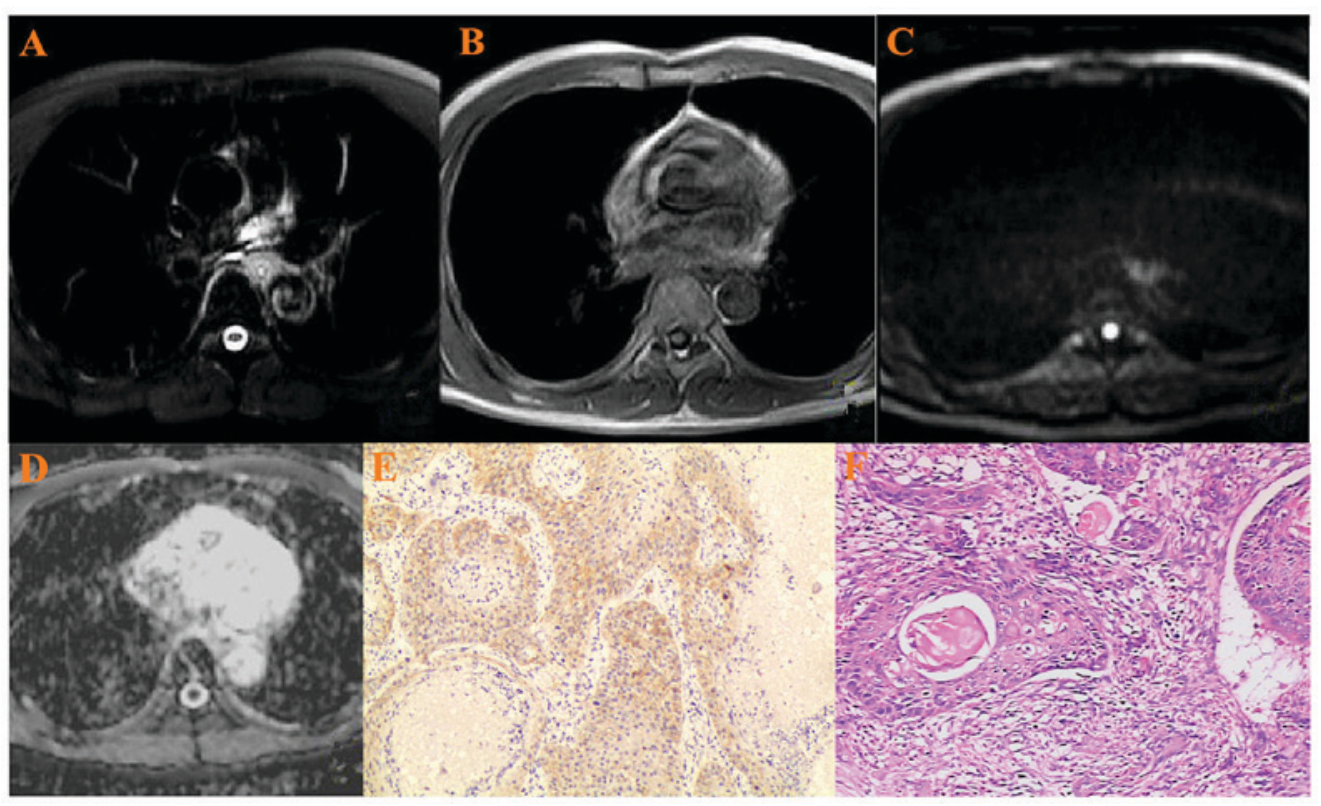

Figure 1. A 75-year-old patient with ESCC in the mid-thoracic portion. (A) ESCC was hyperintense on T2-weighted image, (B) hypointense on T1-weighted image, (C) hyperintense on the DW-MRI $\left(b=800 \mathrm{~s} / \mathrm{mm}^{2}\right)$ (D) hypointense on ADC map. ADC value:1.62 $\pm 0.35 \times 10^{-3} \mathrm{~mm}^{2} / \mathrm{s}$. (E) Low VEGF staining in cytoplasm of ESCC: Weak positive (+) (x200 magnification), (F) Histopathology: Well differentiated squamous cell carcinoma (x200 magnification). ESCC, esophageal squamous cell carcinoma; DW-MRI, diffusion weighted-magnetic resonance imaging; ADC, apparent diffusion coefficient; VEGF, vascular endothelial growth factor.
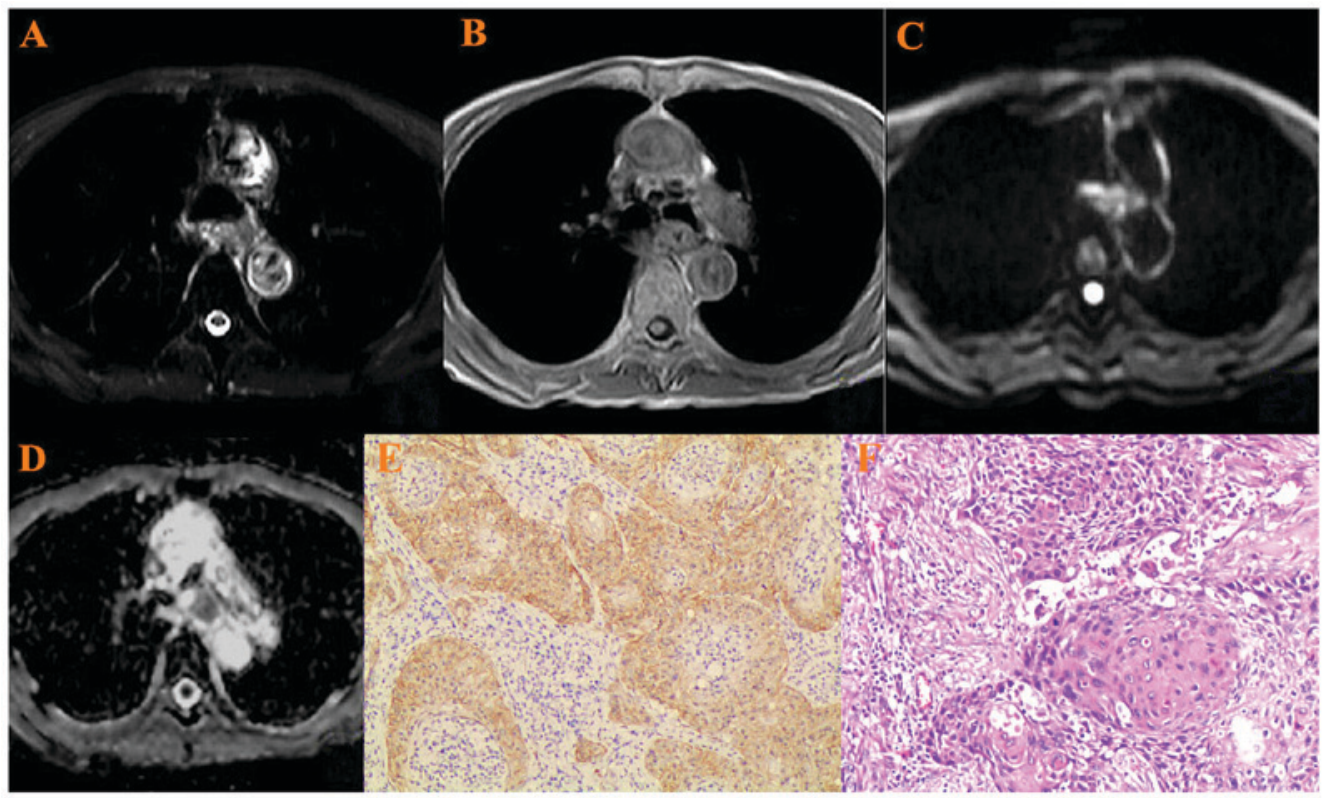

Figure 2. A 68-year-old patient with ESCC in the upper thoracic portion. (A) ESCC was hyperintense on T2-weighted image, (B) hypointense on T1-weighted image, $(\mathrm{C})$ hyperintense on the DW-MRI ( $\left.\mathrm{b}=800 \mathrm{~s} / \mathrm{mm}^{2}\right)$ (D) hypointense on ADC map. ADC value: $1.48 \pm 0.16 \times 10^{-3} \mathrm{~mm}^{2} / \mathrm{s}$. (E) Moderate VEGF staining in cytoplasm of ESCC: Positive (++) (x200 magnification), (F) Histopathology: Moderately differentiated squamous cell carcinoma (x200 magnification). ESCC, esophageal squamous cell carcinoma; DW-MRI, diffusion weighted-magnetic resonance imaging; ADC, apparent diffusion coefficient; VEGF, vascular endothelial growth factor.

correlation if $r$ was a positive value and an inverse correlation if $r$ was a negative value. $\mathrm{P}<0.05$ was considered to indicate a statistically significant difference.

\section{Results}

Correlation of VEGF expression level with MRI features and pathology characters. All 52 esophageal cancer showed hyperintense on T2WI (Figs. 1-3A), hypointense on T1WI (Figs. 1-3B), hyperintense on the DWIs (Figs. 1-3C), hypointense on ADC map (Figs. 1-3D). Two experienced pathologists from The First Affiliated Hospital of China Medical University reviewed the pathological grading independently. There were 10 well-differentiated cancers, 28 moderately differentiated ones and 14 poorly differentiated ones (Figs. 1-3E). VEGF was highly expressed in ESCC with a positive rate of 90.3\%, 


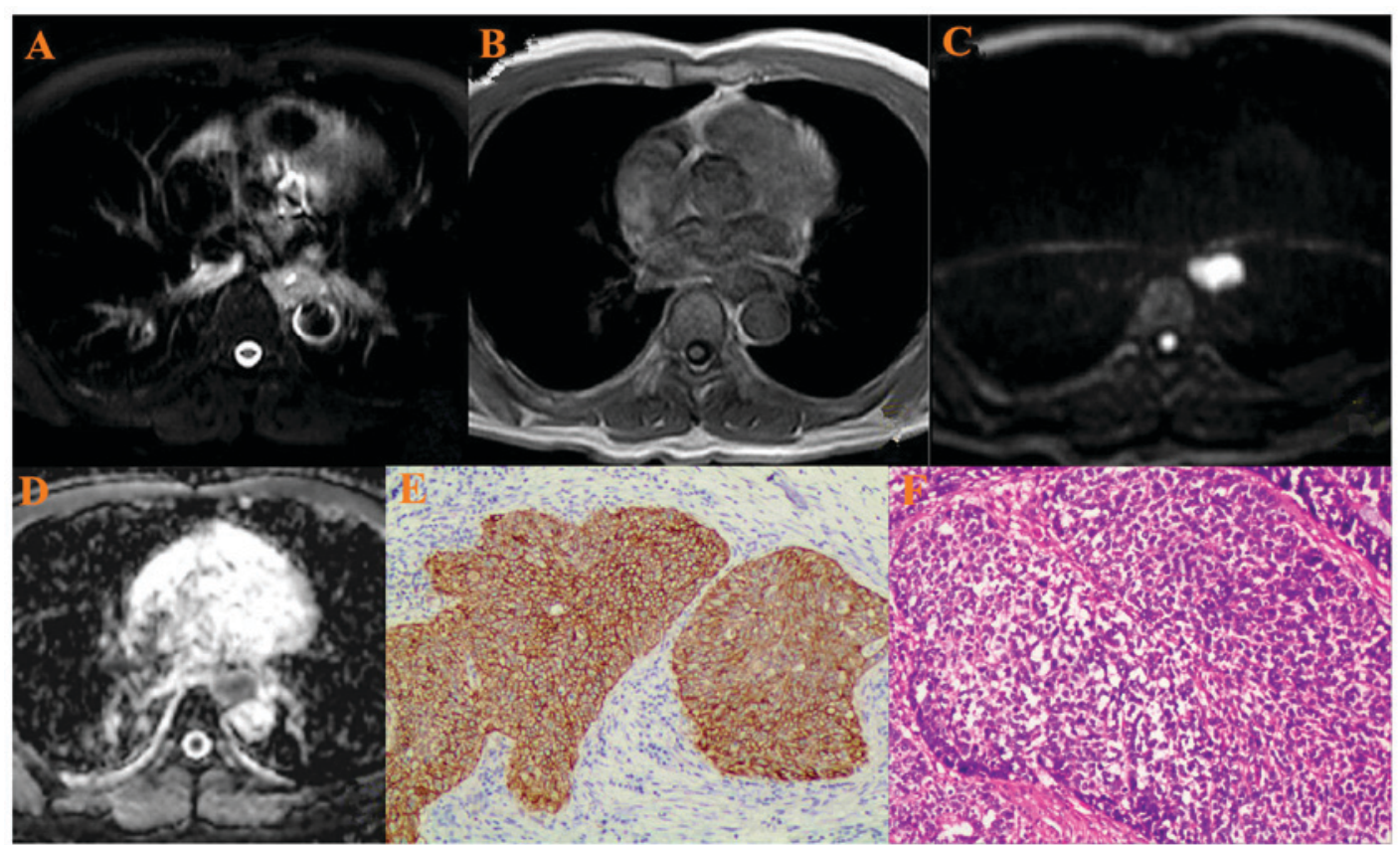

Figure 3. A 72-year-old patient with ESCC in the mid-thoracic portion. (A) ESCC was hyperintense on T2-weighted image, (B) hypointense on T1-weighted image, (C) hyperintense on the DW-MRI ( $\mathrm{b}=800 \mathrm{~s} / \mathrm{mm}^{2}$ ) (D) hypointense on ADC map. ADC value: $1.31 \pm 0.25 \times 10^{-3} \mathrm{~mm} / \mathrm{s}$. (E) High VEGF staining in cytoplasm of ESCC: Strong positive (+++) (x200 magnification), (F) Histopathology: Poorly differentiated squamous cell carcinoma (x200 magnification). ESCC, esophageal squamous cell carcinoma; DW-MRI, diffusion weighted-magnetic resonance imaging; ADC, apparent diffusion coefficient; VEGF, vascular endothelial growth factor.

and weak positive in 5 , positive in 15 and strong positive in 32 samples (Figs. 1-3F). When $b$ was $800 \mathrm{~s} / \mathrm{mm}^{2}$, the mean ADC value in all esophageal cancer was $1.44 \pm 0.32 \times 10^{-3} \mathrm{~mm}^{2} / \mathrm{s}$.

Pathological grading was positively correlated with ADC values in ESCC. When $b$ was $800 \mathrm{~s} / \mathrm{mm}^{2}$, the mean ADC value of the esophageal cancer with different differentiating degree (well-moderately-poorly differentiated) were $1.65 \pm 0.25 \times 10^{-3}, 1.48 \pm 0.31 \times 10^{-3}$ and $1.32 \pm 0.18 \times 10^{-3} \mathrm{~mm}^{2} / \mathrm{s}$, respectively. Variance analysis demonstrated that the difference of ADC value were statistically significant between the three groups $(\mathrm{F}=6.285, \mathrm{P}=0.006)$. LSD post-hoc test demonstrated that there was significant difference between well differentiation and moderately differentiation and poor differentiation; however, no significant difference between moderately differentiation and poor differentiation $(\mathrm{P}=0.005, \mathrm{P}=0.035$ and $\mathrm{P}=0.158$ respectively) was identified . Spearman correlation analysis indicated that the pathological grading of ESCC was positively correlated with ADC values ( $r=0.635, \mathrm{P}=0.0007$ ) (Fig. 4). Therefore, the ADC values may represent the tumor histological differentiation grade.

$V E G F$ expression was inversely correlated with the ADC values in ESCC. When $b$ was $800 \mathrm{~s} / \mathrm{mm}^{2}$, the mean ADC value of the esophageal cancer with VEGF grading (Weakly positive, positive, strongly positive) were $1.45 \pm 0.37 \times 10^{-3}, 1.61 \pm 0.28 \times 10^{-3}$ and $1.27 \pm 0.21 \times 10^{-3} \mathrm{~mm}^{2} / \mathrm{s}$, respectively. Variance analysis demonstrated that the difference of ADC values were statistically significant between the three groups $(F=7.125, \mathrm{P}=0.005)$. An analysis of variance demonstrated that there were no significant differences between VEGF weak and strong positive groups and weak and positive groups $(\mathrm{P}=0.185$ and $\mathrm{P}=0.054)$.

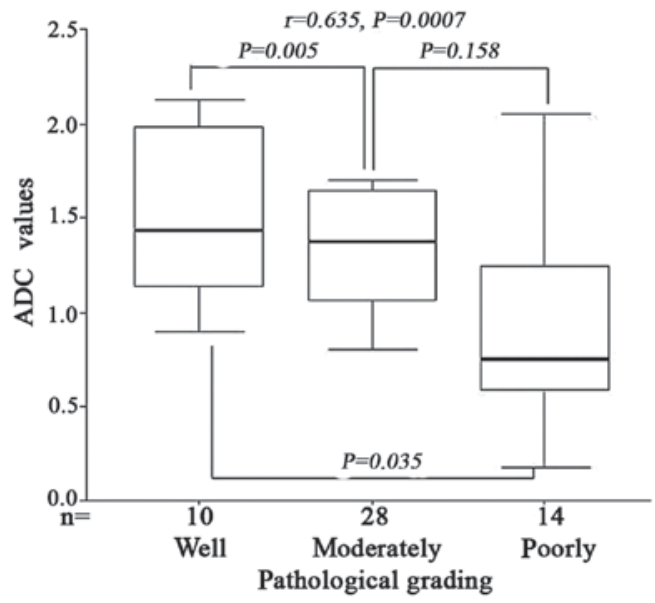

Figure 4. Pathological grading was positively correlated with ADC values in ESCC. There was significant difference between well and moderately differentiation and well and poor differentiation; however no significant difference between moderate and poorly differentiation was identified $(\mathrm{P}=0.158)$. Spearman's correlation analysis indicated that the pathological grading of ESCC was positively correlated with ADC values ( $r=0.635, \mathrm{P}=0.0007)$. ADC, apparent diffusion coefficient; ESCC, esophageal squamous cell carcinoma.

However a significant difference between positive and strong positive groups was identified $(\mathrm{P}=0.003)$. Spearman correlation analysis indicated that VEGF expression was inversely correlated with the ADC values in ESCC ( $r=-0.321, \mathrm{P}=0.008)$ (Fig. 5). Therefore, the ADC values may represent the degree of VEGF expression, and may be an alternative for assessing tumor angiogenesis to predict anti-VEGF response.

Correlation between the pathological grading and VEGF expression in ESCC. Kruskal-Wallis H test demonstrated that 


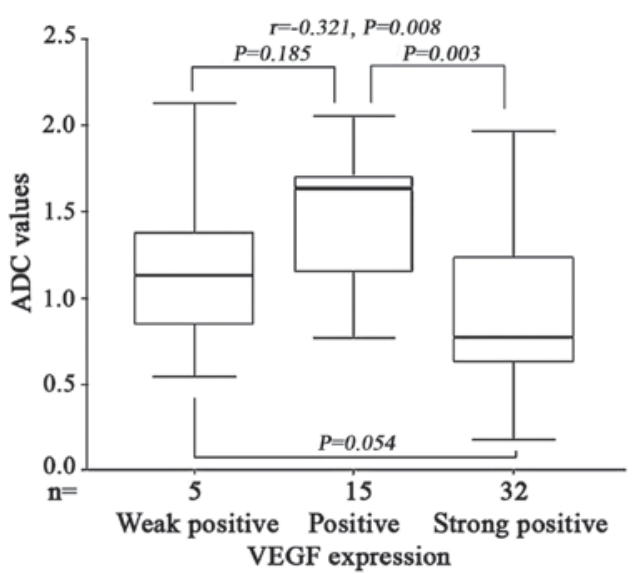

Figure 5. VEGF expression was inversely correlated with the ADC values in ESCC. There was no significant difference between VEGF weak positive and strong positive group, weak positive and positive groups $(\mathrm{P}=0.185)$; however, statistically significant differences were detected between positive and strong positive groups, $(\mathrm{P}=0.054$ and $\mathrm{P}=0.003$, respectively). Spearman correlation analysis indicated that VEGF expression were inversely correlated with the ADC values in ESCC $(r=-0.321, \mathrm{P}=0.008)$. VEGF, vascular endothelial growth factor; ADC, apparent diffusion coefficient.

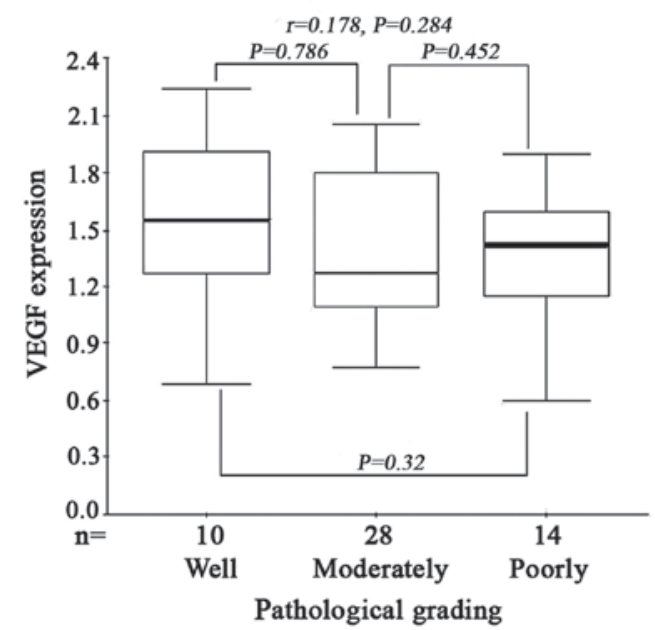

Figure 6. There was no significant difference VEGF expression between well differentiated and moderately differentiated, well differentiated and poorly differentiated, and moderately differentiated and poorly differentiated of VEGF expression $(\mathrm{P}=0.786, \mathrm{P}=0.329, \mathrm{P}=0.452)$. Spearman correlation analysis indicated that there was no correlation between the pathological grading and the VEGF expression in ESCC $(r=0.178, \mathrm{P}=0.284)$. VEGF, vascular endothelial growth factor; ESCC, esophageal squamous cell carcinoma.

there was no significant difference between VEGF expression and pathological grading in ESCC $(H=1.875, \mathrm{P}=0.565)$. No statistical differences were observed between VEGF expression and tumor differentiation $(\mathrm{P}=0.786, \mathrm{P}=0.329, \mathrm{P}=0.452$, between well and moderate, well and poor, and poor and moderate differentiation respectively). Furthermore Spearman's correlation analysis indicated that there was no correlation between the pathological grading and VEGF expression in ESCC $(r=0.178, \mathrm{P}=0.284)$ (Fig. 6).

Changes of ADC values in ESCC prior to and following radiotherapy. All patients were evaluated as CR (30 cases) or PR (22 cases) by endoscopic examination 2 weeks

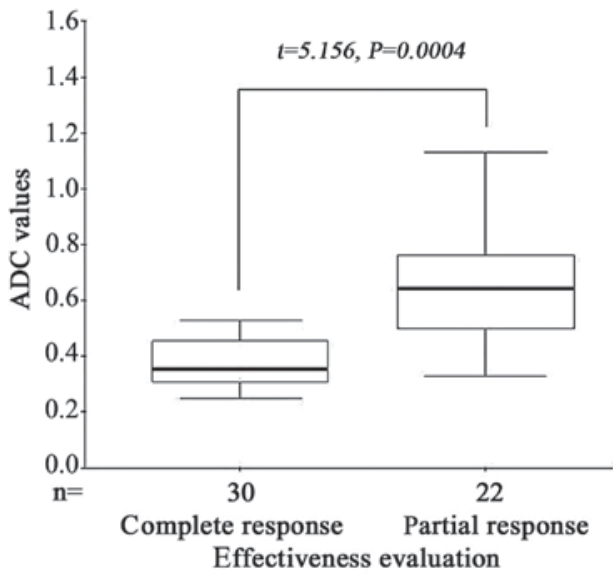

Figure 7. The mean ADC values of the CR group were significantly higher than PR group prior to radiotherapy $(t=5.156, \mathrm{P}=0.0004)$. ADC, apparent diffusion coefficient; $\mathrm{CR}$, complete response; $\mathrm{PR}$, partial response.

following radiotherapy. The mean ADC values of $\mathrm{CR}$ and $\mathrm{PR}$ groups prior to radiotherapy were $1.63 \pm 0.21 \times 10^{-3} \mathrm{~mm}^{2} / \mathrm{s}$ and $1.38 \pm 0.25 \times 10^{-3} \mathrm{~mm}^{2} / \mathrm{s}$, respectively. The mean ADC value prior to radiotherapy of the CR group was significantly higher than the PR group ( $t=5.156, \mathrm{P}=0.0004)$ (Fig. 7). The mean ADC values of $\mathrm{CR}$ and $\mathrm{PR}$ groups following radiotherapy rose to $2.73 \pm 0.28 \times 10^{-3}$ and $1.85 \pm 0.14 \times 10^{-3} \mathrm{~mm}^{2} / \mathrm{s}$, respectively; however no statistical difference was detected between the two groups $(t=0.886, \mathrm{P}=0.356)$. Therefore, ADC values may be a useful marker to predict radiotherapy response. The higher the ADC values, the better the response to radiotherapy, which ultimately improves the therapeutic response and prognosis of the patient.

\section{Discussion}

The development of MRI techniques and the clinical application of DW-MRI, staging and diagnosis have allowed the therapeutic evaluation of tumors to proceed to a higher level (15). The ADC values are obtained by setting the diffusion sensitivity coefficient (b value) (16). ADC value is a quantitative index that reflects the diffusion degree of water molecules (17). Therefore, DW-MRI can noninvasively observe the movement of water molecules in living tissues and provide pathological and physiological changes in vivo (18). Imaging techniques range from observing anatomical changes to functional states. Based on the selection of $b$ value, Baur et al (19) reported that the larger the $b$ value is, the larger the diffusion weight of the image, and the smaller the $\mathrm{T} 2$ penetration effect is, the reduced effect of blood perfusion, and the more accurate the ADC value. Previous studies have reported that, when $b$ was $600 \mathrm{~s} / \mathrm{mm}^{2}$, the DWI exhibited the lesion border with highest credibility compared with the other examined $b$ value, and had the best consistency with pathology $(20,21)$. Taking into account the measured ADC value to accurately reflect the level of water molecules dispersion in tissue, the present study selected $b$ as $800 \mathrm{~s} / \mathrm{mm}$, and the mean ADC values of ESCC obtained by the present study was $1.44 \pm 0.32 \times 10^{-3} \mathrm{~mm}^{2} / \mathrm{s}$. The results are in line with the relevant literature, as several studies have reported that the ADC value is closely associated with the clinical stage, pathological grading, chemoradiotherapy response, and prognosis in gastric, lung, colorectal and liver 
cancer and other solid tumors $(22,23)$. In short, DW-MRI is a reliable noninvasive evaluation method that is being increasingly used in clinical practice.

The present study demonstrated that ADC values were positively correlated with the pathological grading of ESCC. It was also demonstrated that, prior to radiotherapy, the higher the ADC value, the better the radiotherapy response. Therefore, we postulate that ADC values may be used to quantify the pathological grade, radiotherapy response and prognostic indicators of esophageal cancer.

Tumor angiogenesis is a major factor in tumor growth and metastasis (24). Vascular endothelial growth factor (VEGF) serves an important role in the regulation of tumor angiogenesis and differentiation of vascular endothelial cells (17). VEGF can promote the proliferation and differentiation of vascular endothelial cells, and can induce the morphogenesis of capillaries through paracrine way, can stimulate the growth of endothelial progenitor cells (EPCs), which are characterized by high proliferative capacity and neovascularization (25). VEGF and its induced high blood vessel density can promote tumor circulatory metastasis, and VEGF can degrade vascular basement membrane, which creates a suitable microenvironment for circulatory metastasis. VEGF also can promote the formation of new lymphatic vessels in lymph nodes, which leads to an earlier lymph node metastasis (26). Studies have demonstrated that VEGF is overexpressed in gastric, lung, colorectal, breast, prostate, liver cancer and other solid tumors $(27,28)$. Recent studies have reported that the expression of VEGF in esophageal cancer is associated with lymph node metastasis, distant metastasis and clinical stage $(\mathrm{P}<0.05)$, but there is no significant correlation with pathological grading and depth of invasion (29,30). Additionally, overexpression of VEGF suggested poor prognosis of esophageal cancer, a meta-analysis reported that the risk of late stage (III and IV) increased in esophageal cancer patients with VEGF overexpression $(\mathrm{HR}=1.55)$, and the odds ratio (OR) was 2.14 (31). The results of the present study demonstrate that VEGF was highly expressed in ESCC with a positive rate of $90.3 \%$, yet there was no correlation between the pathological grading and the VEGF expression, this result is in accordance with other reported values (32). VEGF has become an important target for anticancer treatments with humanized anti-VEGF monoclonal antibodies such as Bevacizumab being approved by the Food and Drug Administration (FDA), and have proven beneficial to cancer therapy in metastatic colorectal, metastatic breast, advanced non-small cell lung cancer and metastatic renal cell carcinoma (33). In addition, there are some evidences for its challenging efficacy in the treatment of other solid tumors, including hepatocellular carcinoma, gastric and esophageal cancer (34). However, there is still lack of definite biomarkers to predict the anti-VEGF response. VEGF may be useful prognostic biomarker and also a potential marker to predict anti-VEGF response in tumor therapy. However, the clinical application of VEGF is limited by the factors such as trauma, complications and the error of pathological sampling $(35,36)$.

The present retrospective study demonstrated that ADC values were significantly inversely correlated with VEGF expression. Therefore, we hypothesize that ADC values may be a viable alternative for assessing VEGF expression level to predict prognosis and anti-VEGF response.
To conclude, DWI with ADC values measurement may represent the grade of tumor histologic differentiation and the degree of VEGF expression, and also a useful biomarker to predict radiotherapy and anti-VEGF response in ESCC. ADC values may be a substitution for assessing tumor angiogenesis and also a novel prognostic factor and contribute to the treatment of ESCC.

\section{Acknowledgements}

Not applicable.

\section{Funding}

No funding was received.

\section{Availability of data and materials}

The datasets used and/or analyzed during the current study are available from the corresponding author on reasonable request.

\section{Authors' contributions}

QC and GL designed the experiment. QC was a major contributor in writing the manuscript. YW, SZ and $\mathrm{HZ}$ performed the experiments and analyzed the data. All authors read and approved the final manuscript.

\section{Ethics approval and consent to participate}

This study was approved by the Ethics Committee of The First Affiliated Hospital of China Medical University (Shenyang, China). All patients provided written informed consent.

\section{Patient consent for publication}

The study participants provided consent for the data and any associated images to be published.

\section{Competing interests}

The author's declare that they have no conflicts of interest.

\section{References}

1. Yuan X, Wang X, Gu B, Ma Y, Liu Y, Sun M, Kong J, Sun W, Wang H, Zhou F and Gao S: Directional migration in esophageal squamous cell carcinoma (ESCC) is epigenetically regulated by SET nuclear oncogene, a member of the inhibitor of histone acetyltransferase complex. Neoplasia 19: 868-884, 2017.

2. Nakashima S, Shiozaki A, Ichikawa D, Hikami S, Kosuga T, Konishi H, Komatsu S, Fujiwara H, Okamoto K, Kishimoto M, et al: Transient receptor potential melastatin 7 as an independent prognostic factor in human esophageal squamous cell carcinoma. Anticancer Res 37: 1161, 2017.

3. Ojiri H: Diagnostic Imaging of the Esophageal Cancer[M]// Esophageal Squamous Cell Carcinoma. Springer Japan: 33-61, 2015.

4. Gallivanone F, Carne I, Interlenghi M, D'Ambrosio D, Baldi M, Fantinato D and Castiglioni I: A method for manufacturing oncological phantoms for the quantification of 18F-FDG PET and DW-MRI studies. Contrast Media Mol Imaging 2017: 3461684 , 2017. 
5. Kirchner J, Deuschl C, Schweiger B, Herrmann K, Forsting M, Buchbender C, Antoch $G$ and Umutlu L: Imaging children suffering from lymphoma: An evaluation of different ${ }^{18} \mathrm{~F}-\mathrm{FDG}$ PET/MRI protocols compared to whole-body DW-MRI. Eur J Nucl Med Mol Imaging 44: 1742-1750, 2017.

6. Milad P, Elbegiermy M, Shokry T, Mahmoud H, Kamal I, Taha MS and Keriakos N: The added value of pretreatment DW MRI in characterization of salivary glands pathologies. Am J Otolaryngol 38: 13-20, 2017.

7. Weller A, Papoutsaki MV, Waterton JC, Chiti A, Stroobants S, Kuijer J, Blackledge M, Morgan V and deSouza NM: Diffusion-weighted (DW) MRI in lung cancers: ADC test-retest repeatability. Eur Radiol 27: 4552-4562, 2017.

8. Hafeez S, Koh MD, Sohaib A and Huddart R: Use of diffusion weighted-MRI (DW-MRI) as a prognostic biomarker of survival and time to cystectomy in muscle invasive bladder cancer (MIBC) following organ conserving treatment. Eur J Cancer 72: S192, 2017.

9. Misra P, Kirpalani A, Leung G, Vlachou PA, Lee JY, Jothy S, Zaltzman $\mathrm{J}$ and Yuen DA: The role of thrombectomy and diffusion-weighted imaging with MRI in post-transplant renal vein thrombosis: A case report. Bmc Nephrol 18: 224, 2017.

10. Leber MF and Efferth T: Molecular principles of cancer invasion and metastasis (Review). Int J Oncol 34: 881-895, 2009.

11. Jiang JT, Zhang LF, Zhou B, Zhang SQ, Li SM, Zhang W, Zhang J, Qiao Z, Kong RR, Ma YF and Chen S: Relationships of uPA and VEGF expression in esophageal cancer and microvascular density with tumorous invasion and metastasis. Asian Pac J Cancer Prev 13: 3379-3383, 2012.

12. Liu J, Xia J, Zhang Y, Fu M, Gong S and Guo Y: Associations between the expression of MTA1 and VEGF-C in esophageal squamous cell carcinoma with lymph angiogenesis and lymph node metastasis. Oncol Lett 14: 3275-3281, 2017.

13. Joo K, Park SJ, Choi Y, Lee JE, Na YM, Hong HK, Park KH, Kim HM, Chung JY and Woo SJ: Role of the Fc Region in the Vitreous half-life of Anti-VEGF drugs. Invest Ophthalmol Vis Sci 58: 4261-4262, 2017.

14. Mahfouz N, Tahtouh R, Alaaeddine N, El Hajj J, Sarkis R, Hachem R, Raad I and Hilal G: Gastrointestinal cancer cells treatment with bevacizumab activates a VEGF autoregulatory mechanism involving telomerase catalytic subunit hTERT via PI3K-AKT, HIF-1 $\alpha$ and VEGF receptors. PLoS One 12 e0179202, 2017.

15. Huang RW, Chao YK, Wen YW, Chang HK, Tseng CK, Chan SC and Liu YH: Predictors of pathological complete response to neoadjuvant chemoradiotherapy for esophageal squamous cell carcinoma. World J Surg Oncol 12: 170, 2014.

16. Kim CK, Park BK, Lee HM, Kim SS and Kim E: MRI techniques for prediction of local tumor progression after high-intensity focused ultrasonic ablation of prostate cancer. Ajr Am J Roentgenol 190: 1180-1186, 2008.

17. Donati F, Boraschi P, Pacciardi F, Cervelli R, Castagna M, Urbani L, Falaschi F and Caramella D: 3T diffusion-weighted MRI in the response assessment of colorectal liver metastases after chemotherapy: Correlation between ADC value and histological tumour regression grading. Eur J Radiol 91: 57-65, 2017.

18. Bedair R, Priest AN, Patterson AJ, McLean MA, Graves MJ, Manavaki R, Gill AB, Abeyakoon O, Griffiths JR and Gilbert FJ: Assessment of early treatment response to neoadjuvant chemotherapy in breast cancer using non-mono-exponential diffusion models: A feasibility study comparing the baseline and mid-treatment MRI examinations. Eur Radiol 27: 2726-2736 2017.

19. Baur A, Dietrich O and Reiser M: Diffusion-weighted imaging of bone marrow: Current status. Eur Radiol 13: 699-1708, 2003.

20. Tyng CJ, Guimarães MD, Bitencourt AGV, Mattos dos Santos LC Vieira Pinto Barbosa PN, Zurstrassen CE, Nóbrega Pereira E, Gross JL and Chojniak R: Correlation of the ADC values assessed by diffusion-weighted MRI and ${ }^{18} \mathrm{~F}-\mathrm{FDG}$ PET/CT SUV in patients with lung cancer. Applied Cancer Research 38: 9, 2018.
21. Mahmood F, Johannesen HH, Geertsen P and Hansen RH Repeated diffusion MRI reveals earliest time point for stratification of radiotherapy response in brain metastases. Phy Med Biol 62: 2990-3009, 2017

22. Steiger P, Barbieri S, Kruse A, Ith M and Thoeny HC: Selection for biopsy of kidney transplant patients by diffusion-weighted MRI. Eur Radiol 27: 4336-4344, 2017.

23. Yoshida S, Kobayashi S, Koga FJ, Ishioka C, Ishii H, Tanaka Y, Nakanishi Y, Matsuoka N, Numao K, Saito H, et al: The ADC value is a prognostic biomarker of upper urinary tract cancer: Potential application to preoperative risk stratification. Eur Urol Suppl 12: e240-e241, 2013.

24. Pepe P, D'Urso D, Garufi A, Priolo G, Pennisi M, Russo G, Sabini MG, Valastro LM, Galia A and Fraggetta $F$ : Multiparametric MRI apparent diffusion coefficient (ADC) accuracy in diagnosing clinically significant prostate cancer. Vivo 31: 415-418, 2017.

25. Albini A, Bruno A, Noonan DM and Mortara L: Contribution to tumor angiogenesis from innate immune cells within the tumor microenvironment: Implications for immunotherapy. Front Immunol 9: 527, 2018

26. Vora RA: Book review: Anti-VEGF use in ophthalmology. Int J Retina Vitreous 3: 35, 2017.

27. Rafii S and Lyden D: Therapeutic stem and progenitor cell transplantation for organ vascularization and regeneration. Nat Med 9: 702-712, 2003

28. Howell KR and Armstrong J: Vascular endothelial growth factor (VEGF) in neurodevelopmental disorders. Curr Behavioral Neuroscience Rep 9: 1-10, 2017.

29. Okuda T, Tasaki T, Nakata S, Yamashita K, Yoshioka H, Izumoto S, Kato A and Fujita M: Efficacy of combination therapy with MET and VEGF inhibitors for MET-overexpressing glioblastoma. Anticancer Res 37: 3871-3876, 2017.

30. Koh YW, Han JH, Yoon DH, Suh C and Huh J: PD-L1 expression correlates with VEGF and microvessel density in patients with uniformly treated classical Hodgkin lymphoma. Ann Hematol 96: 1833-1890, 2017.

31. Xu XL, Ling ZQ, Chen W, Xu YP and Mao WM: The overexpression of VEGF in esophageal cancer is associated with a more advanced TMN stage: A meta-analysis. Cancer Biomark 13: 105-113, 2013.

32. Tae N, Lee S, Kim O, Park J, Na S and Lee JH: Syntenin promotes VEGF-induced VEGFR2 endocytosis and angiogenesis by increasing ephrin-B2 function in endothelial cells. Oncotarget 8: 38886-38901, 2017.

33. Zhou YL, Chen CL, Wang YX, Tong Y, Fang XL, Li L and Wang ZY: Association between polymorphism rs11200638 in the HTRA1 gene and the response to anti-VEGF treatment of exudative AMD: A meta-analysis. Bmc Ophthalmol 17: 97, 2017.

34. Thulliez M, Angoulvant D, Le Lez ML, et al: Intravitreal anti-VEGF monoclonal antibodies. Biotechnology \& Bioengineering 106: 938-951, 2014.

35. Olivo M: Combined use of anti-VEGF and anti-EGFR monoclonal antibodies with photodynamic therapy suppresses tumor growth in an in vivo tumor model. J Cancer Sci Ther 5: 100-105, 2013.

36. Teng LS, Jin KT, He KF, Zhang J, Wang HH and Cao J: Clinical applications of VEGF-trap (aflibercept) in cancer treatment. J Chin Med Assoc 73: 449-456, 2010.

This work is licensed under a Creative Commons Attribution-NonCommercial-NoDerivatives 4.0 International (CC BY-NC-ND 4.0) License. 